\title{
A 50-year review of minimal extraocular surgery for retinal detachment: five hurdles had to be overcome for its acceptance
}

\author{
Ingrid Kreissig ${ }^{1}$
}

Received: 30 October 2019 / Accepted: 31 October 2019 / Published online: 18 November 2019

(c) The Author(s), under exclusive licence to The Royal College of Ophthalmologists 2019

\begin{abstract}
The non-drainage segmental buckling procedure by Custodis for repair of a retinal detachment harboured serious postoperative complications. To amend this new technique to a minimal extraocular surgery with practically no intra- nor postoperative complications, four hurdles had to be overcome: diathermy was replaced by cryosurgery, polyviol plombe by the silicone sponge, eight rules were defined to find the break in a primary retinal detachment or in an eye up for reoperation and a subsequent 15-year follow-up of anatomical and functional results confirmed that minimal extraocular surgery for repair of retinal detachment suffices for optimal long-term results without harbouring secondary complications threatening regained visual acuity.
\end{abstract}

In 1953 Ernst Custodis from Duesseldorf, Germany introduced a conceptional new technique for treating a retinal detachment. It consisted of: A segmental elastic polyviol plombe and full-thickness diathermy limited to the area of the break and without drainage of subretinal fluid [1]. This new procedure was quickly accepted in Europe and the United States, because there was no longer the risk of serious intraoperative complications that could result after drainage of subretinal fluid.

But in 1960, the enthusiasm for this new procedure abruptly ended due to the reports from the Boston group about serious postoperative complications associated with this new technique including: Endophthalmitis, perforation of the sclera and even enucleation of the treated eye due to the pressure of the polyviol plombe onto sclera that was necrotized after full-thickness diathermy [2]. As a result, this technique was given up in Europe and in the USA. However, not by everybody in the USA, i.e., not by Harvey Lincoff in New York. He had also observed serious postoperative complications but was convinced of the simplicity and rational approach of this new procedure. As a

Ingrid Kreissig

Ingrid.kreissig@medma.uni-heidelberg.de

1 Department of Ophthalmology Mannheim, University of Heidelberg, Heidelberg, Germany consequence, he looked for means to eliminate these deleterious postoperative complications.

The 1st hurdle that had to be overcome was that Lincoff exchanged the not so tissue-inert polyviol plombe by the well-tolerated silicone sponge [3].

The 2nd hurdle, which had to be overcome was that the necrotizing diathermy of the sclera had to be exchanged by another type of coagulation. Though in 1933 Bietti had described the application of cryosurgery, it was not in use then [4]. In 1964 Lincoff et al. introduced cryosurgery as an alternative to diathermy with this new non-drainage procedure $[5,6]$. Yet the question of whether this cryosurgical adhesion is strong enough and as strong as the one after diathermy remained.

To save this non-drainage procedure, Harvey Lincoff looked for proof to confirm that the cryosurgical scar is strong enough. In 1969, I, as a posterior segment surgeon from Germany who had done microscopy as well, came to New York to be trained with Harvey Lincoff. I was most interested in this new atraumatic procedure for repair of a retinal detachment. Thus, I started to work with Harvey Lincoff at New York Hospital, Cornell University and became involved in the new cryosurgery, being questioned for its sufficient retinal adhesiveness. As a consequence, I got involved in extensive 3-year long animal experiments on the adhesiveness of the cryosurgical adhesion on 336 rabbit eyes [7, 8]. By pulling experiments on the cryosurgical adhesion, it could be confirmed that (a) after an ophthalmoscopical control of three different intensities of cryosurgical lesions, three types of 
Fig. 1 The four rules to find the primary break in a retinal detachment. From Kreissig [10]

\section{Rules to Find the Primary Break}
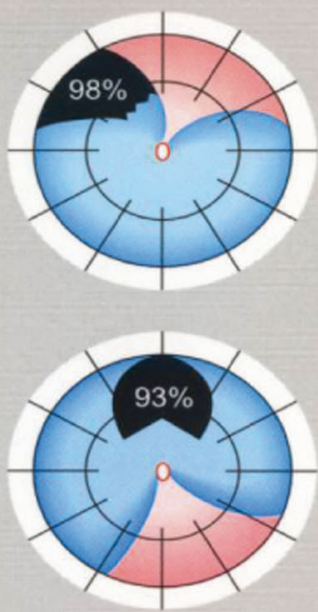

Rule 2:

Total or superior detachments that cross the 12 o'clock meridian: In $93 \%$ the primary break is at 12 o'clock or in a triangle, the apex of which is at the ora serrata, and the sides of which extend $11 / 2$ clock hours to either side of 12 o'clock.

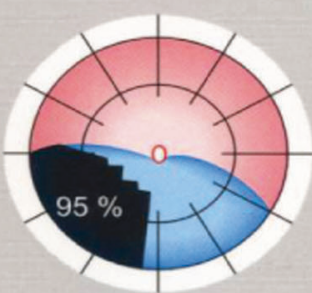

Rule 3:

Inferior detachments: In $95 \%$ the higher side of the detachment indicates on which side of the disc an inferior break lies.

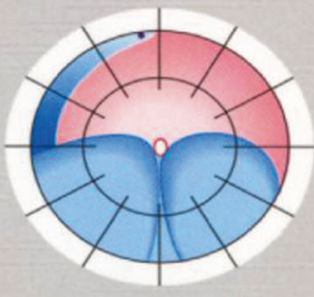

Rule 4:

"Inferior bullous detachment: Inferior bullae in a rhegmatogenous detachment originate from a superior break.

ISBN 3-13-111061-9 (GTV) ISBN 0-86577-781-0 (TNY) adhesive strength could be obtained, (b) that this cryosurgical scar is strong enough as early as after 7 days, and (c) that its adhesiveness continued to increase up to 12 days after application. From then on, these data provided the proof to use cryosurgery instead of diathermy for this new atraumatic non-drainage procedure. However, if not drained intraoperatively, the subretinal fluid will only disappear spontaneously after surgery if the break is found and buckled sufficiently during surgery. Yet often enough this was not the case, and therefore, the retina did not attach after this surgery.

This led to the 3rd hurdle, that is, to find and treat all retinal breaks being present in a detachment to ensure this spontaneous postoperative reattachment. To solve this problem, in 1972 Lincoff and Gieser [9] analysed the preoperative drawings of 1000 retinal detachments and defined the four rules: How to find the retinal break in a primary detachment. These guidelines are in the repertoire of every detachment surgeon, independent of whether the surgery is done as an extraocular or intraocular surgery [10], (Fig. 1).

To apply this atraumatic segmental buckling without drainage as well for eyes that needed a reoperation, in 1996 Lincoff and I analysed the cause of failure in 852 retinal detachments, operated with segmental buckling, cryosurgery and without drainage of subretinal fluid [11]. There were 87 failures: In $76 \%$ - or in about eight of ten failed detachment operations - the cause of failure was either due to a missed retinal break or its insufficient tamponade. As a result, we defined the four rules: How to find the break in an eye up for reoperation [12], (Fig. 2).

By then, every part of the procedure was tried and tested to apply this atraumatic procedure safely for repair of a retinal detachment, which in the meantime was called 
Fig. 2 The four rules to find the missed break in a retinal detachment up for reoperation. From Kreissig [12; back cover]

\section{Rules to Find the Break in Reoperation}

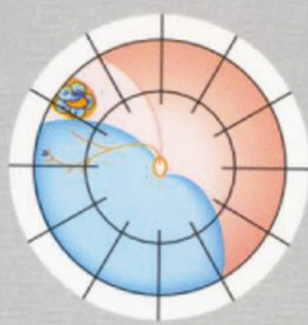

Rule 1:

When the superior border of a temporal or nasal superior detachment drops below the buckle, it implies an undetected break within $11 / 2$ clock hours below the new superior border.

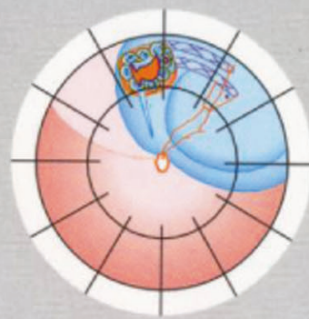

Rule 2:

When the pattern of a detachment (superior, lateral or inferior) converts from one pattern to another, it indicates an undetected break consistent with the new pattern.

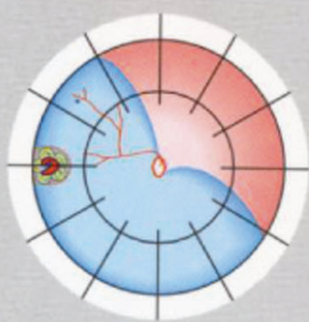

Rule 3:

When the borders of a detachment remain unchanged after a buckling operation and the buckle is in correct position, it implies an undetected break above the buckle.

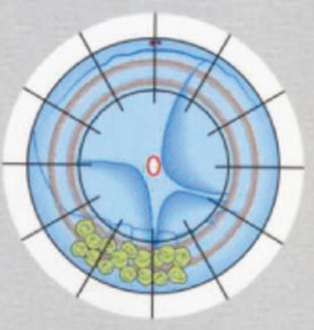

Rule 4:

When a total detachment remains unchanged after being encircled and drained, it implied an undetected break anterior to the existing cerclage near 12 o'clock.

ISBN 3-13-126151-X (GTV)

ISBN 0-86577-994-5 (TNY) minimal segmental buckling or minimal extraocular surgery for repair of retinal detachment [13].

But a 4th hurdle had to be abruptly overcome for final acceptance of this atraumatic procedure because the following question was posed: Does this minimal extraocular surgery, limited to the area of the break, which does not apply a circular buckle, i.e., the cerclage, at all suffice for a long-term retinal attachment? To answer this question, I started an 11-year-postoperative follow-up of 107 consecutive retinal detachments treated with this minimal segmental buckling without drainage between August 1979 and January 1980 [13]. The long-term results were: Primary reattachment in $93 \%$ and after one reoperation, performed without a vitrectomy, in 5 of the 107 operated eyes (4.8\%), reattachment occurred in $97 \%$. During the first postoperative 6 months, the retina redetached in $1 \%$ per month, mostly due to PVR, but after 6 months up to 11 and subsequently up to 15 years postoperatively, the retina redetached only in $0.5 \%$ per year [14]. Thus, if after minimal segmental buckling the retina remained attached up to 6 months postoperatively, the chance for a redetachment up to 15 years is minimal, i.e., it can be almost neglected.

The postoperative complications after this minimal surgery were extraocular and reversible: There were extrusion/ 


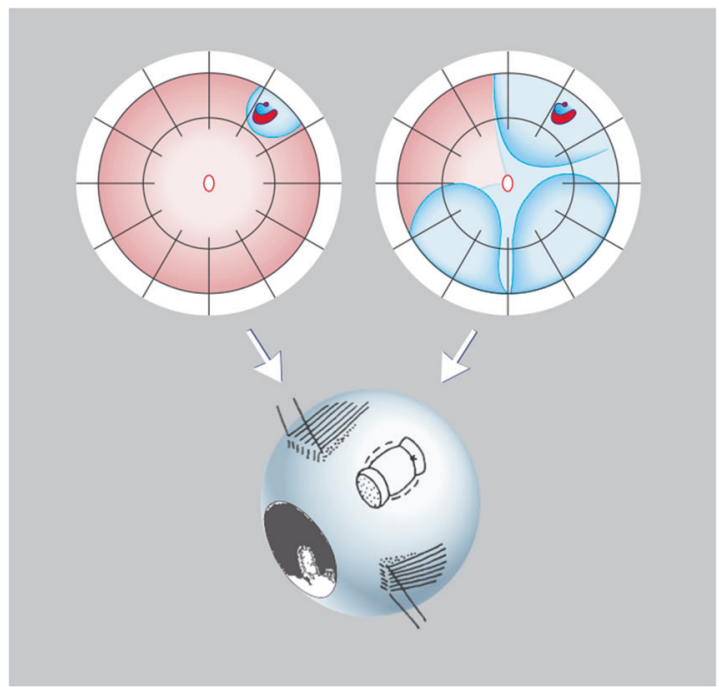

Fig. 3 Minimal segmental buckling without drainage, so-called extraocular minimal surgery: Treatment is limited to area of break and not determined by extent of detachment. The small (top left) and the more extensive detachment (top right) are caused by the same horseshoe tear at 1:00. The treatment of both detachments is the same, consisting of buckling the tear by a segmental sponge, cryosurgery around the break and without drainage of subretinal fluid

infection of the buckle in $0.5 \%$ and diplopia in less than $1 \%$. The cause of final failure was PVR in $3.7 \%$.

In a subsequent meta-analysis of 1854 primary retinal detachments, operated with minimal segmental buckling or a temporary balloon buckle without drainage, the rate of reoperation ranged at $9.1 \%$ and postoperative PVR at $0.9 \%$ [15].

But a 5th hurdle that had to be overcome was, to answer the next question: What will the postoperative visual acuity be after this minimal extraocular procedure? A metaanalysis of 1462 primary retinal detachments, treated by segmental buckling with a sponge or a temporary balloon buckle without drainage, demonstrated a reattachment rate of $97.4 \%$ and a visual acuity of 0.67 or $20 / 30$ after a followup of 2 years [16].

This led to the next question: How will the eye retain the regained visual acuity during a longer follow-up of 15 years. The analysis of the 107 retinal detachments treated by minimal segmental buckling without drainage confirmed the following: The largest increase of visual acuity occurred during the first 6 months after reattachment, but there was still an increase up to 1 year. But from 1 year to 15 years postoperatively there was a slight decrease of 0.07 line per year in the Snellen chart. To find an answer to this, I compared this slight long-term decrease in the operated eye with the visual acuity of the non-operated fellow eye during the 15 years postoperatively. No statistically significant difference in visual acuity was observed between the eye with the buckle in place and the non-operated fellow eye
[14]. This slight decrease in visual acuity over 15 years was comparable with the data of Slataper, who had analysed the development of visual acuity in 17,349 individuals during their life-time [17]. He demonstrated a slight decrease of visual acuity after the age of 60 years and our patients were older than 60 years of age. This slight decrease of visual acuity, observed by Slataper, was actually the same decrease we had observed in our operated and non-operated fellow eyes during the 15-year follow-up.

\section{Conclusion}

Minimal segmental buckling without drainage can be applied in over nine of ten consecutive retinal detachments and be performed under local anaesthesia. The retina remained attached without additional vitrectomy in more than $97 \%$ during a follow-up of 15 years. The rate of reoperation ranged between 4.8 and $9.1 \%$. The regained visual acuity was not jeopardized by secondary complications in comparison with the non-operated fellow during a 15-year follow-up (Fig. 3). Today four options for repair of a retinal detachment are available: Minimal segmental buckling, pneumatic retinopexy, cerclage, and vitrectomy. But whatever procedure is selected for repair of a retinal detachment, still five requirements have to be fulfilled:

(1) The retina has to be attached after one operation.

(2) Performed by a procedure with a minimum of morbidity.

(3) During long-term follow-up the regained visual acuity should not be jeopardized by secondary complications.

(4) The surgery has to be performed under local anesthesia.

(5) On a small budget, since new expensive treatment options have become available for AMD and the resources for ophthalmology are limited.

Therefore, a final conclusion is that less surgery implies less complications and more long-term visual function for the patient.

In this context, minimal segmental buckling with cryosurgery and without drainage of subretinal fluid could fulfil these requirements. One might even refer to the developments in art over the centuries which subsequently resulted in the conclusion:

"Less is More."

\section{Compliance with ethical standards}

Conflict of interest The author declares that she has no conflict of interest. 
Publisher's note Springer Nature remains neutral with regard to jurisdictional claims in published maps and institutional affiliations.

\section{References}

1. Custodis E. Bedeutet die Plombenaufnaehung auf die Sklera einen Fortschritt in der operative Behandlung der Netzhautabloesung? Ber Dtsch Ophth Ges. 1953;58:102-5.

2. Schepens CL, Okamura ID, Brockhurst RJ, et al. Scleral buckling procedures. V. sutures and silicone implants. Arch Ophthalmol. 1960;64:868-81.

3. Lincoff H, Baras I, McLean J. Modifications to the custodis procedure for retinal detachment. Arch Ophthalmol. 1965;73:160-3.

4. Bietti G. Corioretiniti adhesive da crioapplicazioni episclerali. Acta XIV Conc Ophth Madrid. 1933;1:215.

5. Lincoff HA, McLean JM, Nano H. Cryosurgical treatment of retinal detachment. Trans Am Acad Ophthal Otolaryng. 1964;68: 412-32.

6. Lincoff $\mathrm{H}$, Kreissig I. The anatomy of the cryosurgical adhesion. XXI Conc Ophthalmol Mexicana Acta Pars I. 1970;1:406-18.

7. Kreissig I. Ultrastruktur der Kryopexie-Adhaesion in der Netzhautchirurgie (Ultrastructure of the cryopexy adhesion in retinal surgery). Postdoctorial thesis, Germany: University of Bonn; 1972. p 1-172.

8. Kreissig I, Lincoff $\mathrm{H}$. Mechanism of retinal attachment after cryosurgery. Trans Ophthal Soc UK. 1975;95:148-57.
9. Lincoff H, Gieser R. Finding the retinal hole. Arch Ophthalmol 1971;85:565-9.

10. Kreissig I. A practical guide to minimal surgery for retinal detachment. Vol.1. New York: Thieme Stuttgart; 2000. p. 14-15; back cover.

11. Lincoff H, Kreissig I. Extraocular repeat surgery of retinal detachment: a minimal approach. Ophthalmology 1996;103: 1586-92.

12. Kreissig I. A practical guide to minimal surgery for retinal detachment. Vol. 2. New York: Thieme Stuttgart; 2000. p. 320-1; back cover.

13. Kreissig I, Rose D, Jost B. Minimized surgery for retinal detachments with segmental 267 buckling and nondrainage. An 11-year follow-up. Retina. 1992;12:224-33.

14. Kreissig I, Simader F, Fahle M, Lincoff H. Visual acuity after segmental buckling and nondrainage: a 15-year follow-up. Eur J Ophthalmol. 1995;5:240-6.

15. Lincoff H, Lincoff A, Stopa M. Systematic review of efficacy and safety of surgery for primary retinal detachment. In: Kreissig I, editor. Primary retinal detachment: options for repair. Berlin: Springer-Verlag; 2005;8. p. 161-75.

16. Kreissig I Minimal segmental buckling with sponges and balloons for primary retinal detachment. In: Kreissig I, editor. Primary Retinal Detachment: options for repair. Berlin: Springer-Verlag; 2005;6. p. 95-144.

17. Slataper FJ. Age norms of refraction and vision. Arch Ophthalmol. 1950;43:466-79. 